Saudi Journal of Oral and Dental Research

Abbreviated Key Title: Saudi J Oral Dent Res

ISSN 2518-1300 (Print) |ISSN 2518-1297 (Online)

Scholars Middle East Publishers, Dubai, United Arab Emirates

Journal homepage: http://scholarsmepub.com/sjodr/

Review Article

\title{
Correlation of Biological Clock with General and Dental Health
}

Dr. Rishabh Bhanot ${ }^{1 *}$, Dr. Sheikh Javeed Ahmad ${ }^{2}$, Dr. Makrand Sapat, MDS ${ }^{3}$, Dr. N. Vinaya ${ }^{4}$, Dr. Syeda Ayesha ${ }^{5}$, Dr. Deepesh Mathur ${ }^{6}$, Dr. Rahul Vinay Chandra Tiwari ${ }^{7}$

${ }^{1}$ Consultant Oral and Maxillofacial surgeon, SRCJC Hospital, Ludhiana

${ }^{2}$ Assistant professor, Department of Medicine, King Khalid University, Abha, KSA

${ }^{3}$ Consultant Prosthodontist, Ravi Nagar Wani, Yavatmal, Maharashtra India

${ }^{4}$ P.G in Oral pathology, Drs .Sudha and Nageswara Rao Siddhartha institute of dental sciences, Chinoutpalli, Gannavaram Mandal, Andhra Pradesh India

${ }^{5}$ Intern, Sri Sai College of Dental Surgery, Vikarabad, TS, Telangana India

${ }^{6}$ BDS (MBA), Admin, FMS Dental Hospitals, Hyderabad, Telangana, India

${ }^{7}$ FOGS, MDS, Assistant Professor, Department of Oral and Maxillofacial Surgery, Sri Sai College of Dental Surgery, Vikarabad, Telangana India

DOI: $\underline{\text { 10.36348/SJODR.2019.v04i12.003 }}$ | Received: 26.11.2019| Accepted: 02.12.2019| Published: 09.12.2019

*Corresponding author: Dr. Rishabh Bhanot

\section{Abstract}

Most of the physiological processes in the mammals exhibit the circadian rhythms which are guided by endogenous biological clock. This biological clock consists of a central unit which is located in hypothalamic suprachiasmatic nucleus $(\mathrm{SCN})$ and the secondary clocks are located in the peripheral tissues. Circadian rhythms endure 24-hour oscillations of substantial number of the master genes that control the synchronization of varied metabolic and physiological actions within our bodies. This is a very complex regulative network which contributes to an important association between brain and several peripheral tissues as well as organs. The circadian fluctuations of the gene expression are regulated at the molecular level by a family of transcription factors known as "clock genes". The imbalance or dysregulation in the expression of these clock genes can result in the varied human pathological conditions, inclusive of cancer and autoimmune diseases. The evidences are escalating regarding the effect of circadian clock in the tooth development, homeostasis of the oral epithelium, salivary gland and saliva production. The present article summarizes the available knowledge regarding the roles of the clock genes in the genesis as well as perpetuation of the oral tissues, and also mention about the potential association between the "oral clocks" and the diseases like head and neck cancer as well as Sjögren's syndrome.

Keywords: Biological clock, circadian rhythm, clock genes.

Copyright @ 2019: This is an open-access article distributed under the terms of the Creative Commons Attribution license which permits unrestricted use, distribution, and reproduction in any medium for non-commercial use (NonCommercial, or CC-BY-NC) provided the original author and source are credited.

\section{INTRODUCTION}

In order to survive and thrive in constantly changing environment, living beings have a great ability to adapt which is an important selective advantage. One of these abilities is the circadian clock, which has been organized by the living beings in order to maintain the synchronicity of internal mechanisms as well as their daily behavior with the most intense environment signal: The circadian life cycle of 24hours. The biological rhythms can be produced inside an organism by endogenous biological clocks, or guided by the cyclic incidents in the environment like the dark/ light cycle. The circadian rhythms are controlled by the body's biological clocks. The central or the "master" clock is located in the hypothalamus, which is called as the suprachiasmatic nucleus $(\mathrm{SCN})$ while periphery or subordinate clocks are located in the other parts of the body. The central clock is light-responsive and driven by the light/dark cycles. Moreover, the peripheral clocks cannot recognize the light but can be regulated by the central clock or it can also act independently by the other physiological stimuli like feeding. However, the mechanism of the interactions by which the central clock regulates the peripheral clocks is ambiguous. The circadian rhythms are the behavioral, physical and mental changes that are tagged along with a daily cycle. Primarily they respond to the light and darkness in an organism's environment. An example of light related circadian rhythm is the sleeping at night while being awake during the day time. The circadian rhythms are developed in most of the living things inclusive of the plant, animals and many microorganisms. Chronobiology is the study of these circadian rhythms. The biological clocks are intrinsic or innate timing device of an organism. These are composed of various specific molecules (mostly proteins) throughout the 
body which interact within the cells. The biological clocks are found in nearly every tissue as well organ. The researchers have also identified the similar genes in people, mice, fungi, fruit flies as well as several other organisms that are responsible for the production of the components of the clock. The circadian rhythms are related to the sleep and help in determining our sleep patterns. The master clock of the body or suprachiasmatic nucleus $(\mathrm{SCN})$ controls the production of a hormone named melatonin that makes us sleepy. It receives the information about the incoming light from the optic nerves, which transmit this information from eyes to the brain. When there is less amount of light, like in the night, the SCN communicate with the brain to make more of the melatonin in order to get drowsiness.

\section{The biological clock and general health \\ Various epidemiological studies have} implicated the circadian clock as a factor in varied number of diseases which showed a high incidence of cancer in the people who works for long shifts [1]. The hypothesis light-at-night (LAN) has set down the hormone melatonin in the centre of the cancer disease process. According to this hypothesis, melatonin holds the role beyond being just a hormone; it is an important scavenger of the reactive oxygen species. The melatonin is primarily produced at night and gets suppressed by the light; thereby the hypothesis argues that when the people are exposed to the light at night, the oncogenesis is more likely to result. There is strong epidemiological corroboration for the LAN- breast cancer connection [2]. It is also noted that there is less chance for occurrence of the breast cancer in the totally blind people than sighted controls[3]. However, the fact that living against the circadian clock is associated with many melatonin-unrelated pathologies have suggested many additional aetiologies which are linking to the cancer and work shift.However, this idea has been supported by various recent shift work experiments in the mice that do not express the melatonin and show the exacerbated tumour formation[4]. Sleep deprivation and the misalignment of the circadian cycle often develop due to out of the timing of sleep. The only clock-related health condition classified by the ICD-10 (International Statistical Classification of Diseases and Related Health Problems, 10th edition) refers to the 'Disorders of the sleep wake schedule'. There has been found a lack of the consolidated sleep in the patients having neurodegenerative disorders like Alzheimer's, Parkinson's and Huntington's diseases or the more general classification of dementia. Some of this lack may be due to the disease-associated pathology $[5,6]$, but there are also indications that the low amplitude light- dark cycles also contribute to this condition ${ }^{7}$ Seasonal Affective Disorder (SAD), sometimes also called winter depression has also been linked to the circadian clock[8]. Like most of the autoimmune diseases, various pathways of immune dysregulation has been implicated in the rheumatoid arthritis (RA) along with the genetic and environmental associations, which causes both the joint-based and the systemic inflammatory disease. Many of these pathways are generally regulated by the circadian clock, which includes the pro-inflammatory cytokine such as IL-6, expression of the tumor necrosis factor- $\alpha$ (TNF- $\alpha$ ), regulation of transcription of various peroxisome proliferator activated receptor (PPAR) subtype genes and also the activity of the glucocorticoid receptor. Thereby, it is reasonable to hypothesize that apprehension in the expression of the clock gene may contribute to the multiple downstream immunologic effects as seen in complex autoimmune disease like RA and other co-existing autoimmune disease like Sjögren's syndrome (SS)[9].

\section{The Biological Clock and Oral Health}

The teeth are mainly composed of 3 mineralized tissues i.e. enamel, dentin and cementum. Various observations have strongly suggested that the mineralization of the dental tissue is controlled by the complex biological clocks. As a matter of fact, the enamel, dentin, and cementum are formed by the additive modes of growth that preserve the short and long periodical lines within the hard tissues. The information comprised in these periodic lines is increasingly being used in many fields such as evolutionary biology, forensics, anthropology, archaeology and dental development. Complete and appropriate utilization of the information which are recorded in the mineralized dental tissues requires the understanding in the formative as well as circadian controls aspect that causes the teeth to develop as they do. Assimilating the circadian controls that control the tooth shape permits the characterization and the identification of the biological regulatory pathways that dictate the differentiation of the dental cell.

\section{Oral Epithelium}

In several epithelial craniofacial tissues, the clock gene expression has been detected, primarily in the basal cells of the oral epithelium, including the juctional and palatal epithelia and also in the epithelial rests of Malassez surrounding the dental roots. The role of the clock gene expression in oral epithelium yet to be elucidated [10].

\section{Salivary glands}

It has been investigated in various studies that the circadian clock of the salivary flow rate follows the circadian rhythms. The evidence suggests that the salivary secretion follows the circadian rhythms which are important for the self-defense and the effective nutrition. The studies suggest that the salivary glands (SGs) may accommodate a circadian clock in order to regulate the amount, type and the content of saliva. In fact, such circadian clock mechanism has also been demonstrated in other vital organs such as kidney. Kidney and the salivary glands share the several physiological mechanisms which includes a great 
number of water and ion channel activities. Multiple genes that regulate the fluid movement are highly expressed in both the salivary glands as well as the kidney. It has been recently shown in experiments that the clock genes are expressed in SG, similar to the kidney, supporting the idea of a peripheral clock in SGs. It has also been demonstrated that the clock genes regulate the gene for water channel i.e., aquaporin-5 (Aqp5). This gene plays a crucial role in the salivary fluid secretion, advocating the fact that any abnormal expression of the clock genes will affect the expression of Aqp5, resulting in the changes in salivary flow. Some observations also suggest that the alteration in the circadian clock is also responsible for the reduced saliva in person with Sjogren 's syndrome (not yet published observation). Further studies are required in order to establish the appropriate diagnostic tools and also to provide a basis for the innovative treatment options for the patients with SS as well as others.

\section{Oral and Head and Neck Cancer}

Most of the cellular processes are controlled by the circadian clock, including the cell proliferation. Due to changes in the lifestyle in the industrialized societies, $50 \%$ of the human population experiences the frequent disruption of the endogenous circadian rhythm, which can also contribute to the increased development of cancer worldwide. The recent literature suggests that the clock genes play a key role in cancer progression in humans. The expression of some critical cancer-related genes, like p-53 and c-myc has been reported to follow an endogenous circadian rhythm. The loss of the function of circadian genes leads to deregulated neoplastic growth and DNA damage, which results in the uncontrolled cell proliferation, oncogenic activation and increased tumor development in the mice.

\section{CONCLUSION}

Explication of the circadian clock mechanism assures a better understanding of the oral tissue formation. This also opens an interesting new area for the research which can link the systemic and the oral health. It is still not very clear about what regulates the oral circadian clocks. In addition to the previously known circadian clock outputs, it can now be postulated that the saliva production and its contents as well as mineralized tissue formation might be the valid outputs of the peripheral circadian clocks. However, it is unclear at this point also that how the different outputs are influencing the general communication between the central and the peripheral clocks and thereby regulating the whole body as a system.

\section{REFERENCES}

1. Tynes, T., Hannevik, M., Andersen, A., Vistnes, A.I., \& Haldorsen, T. Incidence of breast cancer in Norwegian female radio and telegraph operators. (1996). Cancer Causes Control. 7; 197-204.

2. Hansen, J.(2001). Light at night, shiftwork, \& breast cancer risk. J. Natl. Cancer Inst, 93, 15131515.

3. Flynn-Evans, E.E., Stevens, R.G., Tabandeh, H., Schernhammer, E.S., \& Lockley, S.W.(2009). Total visual blindness is protective against breast cancer. Cancer Causes Control 20, 1753-1756.

4. Van Dycke, K.C., Rodenburg, W., van Oostrom, C.T., VAN Kerkhof, L.W., Pennings, J.L., Roenneberg, T., van Steeg, H., and van der Horst, G.T. (2015). Chronically alternating light cycles increase breast cancer risk in mice. Curr. Biol; 25 , 1932-1937.

5. Coogan, A.N., Schutova, B., Husung, S., Furczyk, K., Baune, B.T., Kropp, P., Hassler, F., \& Thome, J. (2013). The circadian system in Alzheimer's disease: disturbances, mechanisms, and opportunities. Biol. Psychiatry, 74, 333-339.

6. Morton, A.J., Wood, N.I., Hastings, M.H., Hurelbrink, C., Barker, R.A., \& Maywood, E.S. (2005). Disintegration of the sleep-wake cycle and circadian timing in Huntington's disease. J. Neurosci; 25, 157-163.

7. Riemersma-van der Lek, R.F., Swaab, D.F., Twisk, J., Hol, E.M., Hoogendijk, W.J., \& Van Someren, E.J.(2008). Effect of bright light and melatonin on cognitive and noncognitive function in elderly residents of group care facilities: a randomized controlled trial. JAMA. 299, 2642-2655.

8. Bechtel, W. (2015). Circadian rhythms and mood disorders: are the phenomena and mechanisms causally related? Front. Psychiatry, 6118.

9. Athanassiou-Papaefthymiou, M., Kim, D., Harbron, L., Papagerakis, S., Schnell, S., Harada H. (2011). Molecular and circadian controls of ameloblasts. Eur J Oral Sci. 119(Suppl 1):35-40.

10. Zheng, L., Seon, Y.J., Mourao, M.A., Schnell, S., Kim, D., Harada, H. (2013). Circadian rhythms regulate amelogenesis. Bone, 55:158-165. 